\title{
Propiedades psicométricas del Inventario de Hábitos de Estudio CASM-85: un estudio multicéntrico con estudiantes de secundaria peruanos
}

\author{
Psychometric properties of the Inventory of Study Habits CASM-85: a multicenter study \\ with Peruvian high school students
}

\author{
Joel Figueroa-Quiñones ${ }^{\mathrm{a}}$, Juan Walter Pomahuacre Carhuayal ${ }^{\mathrm{b}}$, Yuri Chávez ${ }^{\mathrm{c}}$, Heber Gómez $^{\mathrm{a}, \mathrm{b}}$, \\ Roxana Portocarrero ${ }^{\mathrm{a}}$, Giulianna Gamero-Vega ${ }^{\mathrm{a}}$, Ada Calderón-Giraldo ${ }^{\mathrm{b}}$, Elizabeth López ${ }^{\mathrm{b}}$, \\ Martín Castro $^{\mathrm{b}}$, Juan Carlos Bazo-Alvarez ${ }^{\mathrm{a}, \mathrm{b}, *}$ \\ ${ }^{a}$ Instituto de Investigación, Universidad Católica los Ángeles de Chimbote, Chimbote, Perú. \\ ${ }^{\mathrm{b}}$ Instituto de Investigación, Capacitación y Desarrollo Psicosocial y Educativo (PSYCOPERU), Lima, Perú. \\ ${ }^{c}$ Escuela Profesional de Psicología, Universidad Nacional de San Agustín de Arequipa, Perú.
}

Recibido: 20 de febrero de 2019

Aceptado: 01 de noviembre de 2019

\section{Resumen}

Objetivo: evaluar la estructura interna, invarianza de medida, validez de criterio y confiabilidad del CASM-85 en estudiantes de secundaria peruanos. Materiales y Métodos: se evaluaron 2075 estudiantes de ambos sexos, entre 11 y 18 años, provenientes de colegios nacionales y particulares de Arequipa, Chimbote, Lima y Pucallpa. Se realizó un análisis de la correlación ítem-ítem e ítemresto para cada dimensión del CASM-85, un análisis factorial confirmatorio y modelos MIMIC (Multiple Indicator, Multiple Cause) para evaluar la invarianza de medida a través de grupos según sexo, edad, colegio y ciudad. La confiabilidad fue estimada mediante los coeficientes Alpha y Omega. La validez de criterio entre el CASM85 y el rendimiento académico se revisó a través de la $r$ de Pearson y modelos de regresión. Resultados: se eliminaron ítems heterogéneos y se obtuvo una forma reducida de 45 ítems a compararse con su original de 53 ítems. Para la forma original el CFI y TLI fue $<.9$ en todas las ciudades, mientras que para la forma reducida fue $<.9$ solo en Chimbote. Los SRMR y RMSEA fueron $\leq .10 \mathrm{y} \leq .09$ para las formas original y reducida respectivamente. Los modelos MIMIC confirmaron invarianza para todas las variables excepto para la variable ciudad con la forma original $(|\Delta \mathrm{CFI}|=.09,|\Delta \mathrm{TLI}|=.08)$. La confiabilidad fue moderada tanto para la forma original $(\Omega \geq .68 ; \alpha \geq .81)$ como para la reducida $(\Omega \geq .77 ; \alpha \geq .84)$. La $r$ de Pearson mostró una relación positiva y de fuerza débil para la forma original $(r=.17 ; p<.001)$ y reducida $(r=.18 ; p<.001)$. Conclusión: la forma reducida del CASM-85 (45 ítems) ha mostrado mejores propiedades psicométricas que su forma original.

Palabras clave: hábitos de estudio, CASM-85, propiedades psicométricas, modelos MIMIC.

\section{Para citar este artículo:}

Figueroa-Quiñones, J., Pomahuacre, J. W., Chávez, Y., Gómez, H., Portocarrero, R., Gamero-Vega, G., Calderón-Giraldo, A., López, E., Castro, M., \& Bazo-Alvarez, J. M. (2019). Propiedades psicométricas del Inventario de Hábitos de Estudio CASM-85: un estudio multicéntrico con estudiantes de secundaria peruanos. Liberabit, 25(2), 139-158. https://doi.org/10.24265/liberabit.2019.v25n2.02

\section{Abstract}

Objective: To assess the internal structure, measurement invariance, criterion validity and reliability of the CASM- 85 in Peruvian high school students. Materials and methods: Two thousand seventy-five (2075) students of both sexes, aged 11 to 18 years, from public and private high schools of Arequipa, Chimbote, Lima and Pucallpa were evaluated. An item-item and item-rest correlation analysis for each dimension of the CASM-85, a confirmatory factorial analysis and MIMIC (Multiple Indicator, Multiple Cause) models were used to evaluate the measurement invariance across the groups according to their sex, age, school and city. Reliability was estimated using alpha and omega coefficients. The criterion validity between the CASM- 85 and academic performance was determined using Pearson's $r$ and regression models. Results: The heterogeneous items were removed, obtaining a 45-item short form compared to the original 53-item form. In the original form, CFI and TLI were $<.9$ in all the cities, whereas in the short form, CFI and TLI were $<.9$ only in Chimbote. SRMR and RMSEA were $\leq .10$ and $\leq .09$ in the original and short forms, respectively. The MIMIC models confirmed the measurement invariance in all variables, with the exception of the original form between cities $(|\Delta \mathrm{CFI}|=.09,|\Delta \mathrm{TLI}|=.08)$. Reliability was moderate for both the original $(\Omega \geq .68 ; \alpha \geq .81)$ and short forms $(\Omega \geq .77$; $\alpha \geq$.84). Pearson's $r$ showed a positive and weak correlation in the original $(r=.17, p<.001)$ and short $(r=.18, p<.001)$ forms. Conclusion: The CASM- 85 short form ( 45 items) has shown better psychometric properties than its original full form.

Keywords: study habits, CASM-85, psychometric properties, MIMIC models.

Este es un artículo Open Access bajo la licencia Creative Commons Atribución-NoComercial-Compartirlgual 4.0

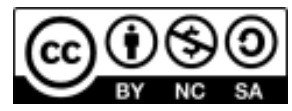

Universidad de San Martín de Porres, Lima - Perú http://ojs3.revistaliberabit.com 


\section{Introducción}

Los hábitos de estudio son el conjunto de conductas rutinarias empleadas para estudiar (Vicuña, 1998). En general, los hábitos pueden categorizarse o dividirse en tipos como 1) la forma de organizarse para estudiar (e.g., tomar apuntes de la clase, o repasos mediante lecturas o subrayados de palabras del texto que se lee), 2) las estrategias usadas para la resolución de las tareas (e.g., buscar comprender el significado de palabras desconocidas, o procurar ejecutar las tareas en un tiempo determinado), 3) la manera de prepararse ante un examen (e.g., el tiempo que se invierte para estudiar), 4) la forma en cómo el estudiante escucha durante las horas de clase (e.g., mantenerse atento o distraerse fácilmente en clases), y 5) la manera en cómo este estudia en casa (e.g., procurar reducir los elementos distractores durante los momentos de estudio como la música, conversaciones con los padres o amigos, entre otros) (Vicuña, 2005). Los hábitos pueden ser considerados positivos si contribuyen efectivamente al aprendizaje y, con ello, a un buen rendimiento académico; caso contrario, los hábitos son vistos como negativos y una intervención suele ser deseable (Vicuña, 1998, 2014).

Existe evidencia acumulada que indica que los hábitos de estudio son un factor clave entre aquellos que determinan el rendimiento académico (Manuel, Grewal, \& Lee, 2018). En principio, sabemos que adecuadas técnicas y estrategias de estudio optimizan la adquisición de conocimientos y el aprendizaje (Alzahrani, Soo-Park, \& Tekian, 2018). Por ejemplo, estudiar con suficiente anticipación y hacer repasos constantes permite que el estudiante pueda asimilar, recuperar, entender y luego usar la información a voluntad (Larose, Bernier, \& Tarabulsy, 2005; Vicuña, 1998; West \& Sadoski, 2011), hábitos que implican saber gestionar el tiempo adecuadamente. De hecho, otros estudios refieren que es posible mejorar el aprendizaje y, en consecuencia, el promedio en las calificaciones si los estudiantes hacen uso adecuado del tiempo a la vez que se enfocan en mejorar su forma de estudiar (Al Shawwa et al., 2015; Salem et al., 2013; Ubaka, Sansgiry, \& Ukwe, 2015; Vicuña, 2005). Estas estrategias utilizadas para mejorar el aprendizaje no solo favorecen la adquisición de conocimientos, sino también las habilidades que componen las competencias que se pretende desarrollar en el estudiante (Villardón, 2006). Por el contrario, una inadecuada organización del tiempo para estudiar (e.g., cómo estudiar de manera apresurada antes de un examen) genera que el estudiante memorice información sin entenderla y/o solo para recordarla por periodos muy breves, afectando con ello el propósito mismo de las clases o lecciones (Bickerdike, O'Deasmhunaigh, O'Flynn, \& O'Tuathaigh, 2016; Diseth, Pallesen, Brunborg, \& Larsen, 2010). Asimismo, estudiar un breve tiempo con constantes distracciones no permite desarrollar la concentración necesaria, luego la información estudiada no se conceptualiza adecuadamente, obteniéndose como resultado serias dificultades para recordar la información (Mira \& López, 1995) e integrarla con información previa o nueva o, más aun, con habilidades específicas que requieren utilizar dicha información perjudicando con ello el aprendizaje.

El rendimiento académico, a su vez, es un indicador importante que guarda una estrecha relación con el éxito o fracaso escolar (Sze, 2017). El éxito escolar es reflejado por los estudiantes que logran adquirir, al menos, las competencias mínimas esperadas durante determinados periodos de estudio. Por el contrario, si un estudiante no concluye los estudios, o persiste en sucesivas repeticiones o suspensiones, se considera que ha fracasado en la escuela (Enguita, Martínez, \& Gómez, 2010; Marchesi, 2003). El estudiante que aprende a resolver de manera satisfactoria actividades académicas como exámenes, exposiciones, entre otros, obtiene un buen rendimiento académico (Mira \& López, 1995); lo que se traduce habitualmente en aprendizaje efectivo y éxito escolar. Así, el rendimiento académico alto es generalmente un buen indicador para predecir si el estudiante logrará el éxito escolar deseado (Mira \& López, 1995; Vicuña, 1998). 
Los hábitos de estudio se pueden medir a través de múltiples pruebas estandarizadas. Entre las más populares en el habla inglesa se encuentran «Survey of Study Habits and Attitudes (SSHA)», «Learning and Study Skills Inventory (LASSI)», "Inventory of Learning Processes (ILP)» y «Study Attitudes and Methods Survey (SAMS)» (Credé \& Kuncel, 2008). En algunos países de Latinoamérica como Argentina, Chile, México o Guatemala, dichos instrumentos vienen siendo revisados y utilizados en el ámbito educativo e investigativo (Bruna, Pérez, Bustos, \& Núñez, 2017; Freiberg, Ledesma, \& Fernández, 2017; Osés, Aguayo, Duarte, \& Manuel, 2010; Stover, Uriel, \& Fernández, 2012). Estos instrumentos coinciden en revisar la forma, estrategias y métodos del estudiante para realizar sus actividades académicas. Particularmente, instrumentos como el SSHA y LASSI han sido validados mediante su contraste con el rendimiento académico, mostrando ser buenos predictores del mismo (Credé \& Kuncel, 2008; Larose et al., 2005; Vicuña, 1998).

En Perú, los usuarios habitualmente muestran preferencia por el Inventario de Hábitos de Estudio CASM-85, un instrumento de gesta local que presenta muchas similitudes con los otros instrumentos ya mencionados. El Inventario de Hábitos de Estudio CASM-85 fue elaborado en 1985 por el psicólogo peruano Luis Vicuña Peri, revisado en 1998 y reimpreso en el 2005 y 2014 por el mismo autor (Vicuña, 1985, 1998, 2005, 2014). Fue diseñado como un instrumento de aplicación grupal o individual, especialmente dirigido a estudiantes de educación secundaria y del primer año de educación superior (Vicuña, 2005). El CASM-85 ha sido estandarizado por su autor únicamente en población de Lima (Vicuña, 1998, 2005, 2014); no obstante, dos estudios independientes han tratado de confirmar su validez y confiabilidad en estudiantes de dos ciudades vecinas de la sierra norte del país. En Huamachuco (La Libertad, Perú), un estudio realizado con 1002 estudiantes de secundaria reportó una confiabilidad aceptable $(\Omega>.70)$ y confirmó la estructura de 5 dimensiones reportada por el autor en población limeña (Armas, 2017). En Otuzco (La Libertad, Perú), otro estudio realizado con 467 adolescentes de una institución educativa reportó una alta consistencia interna $(\mathrm{KR}-20>$.85) y similar validez de estructura interna (Rebaza, 2016). A pesar del aporte de estos estudios -ambos realizados en la sierra norte peruanaes importante resaltar que aún no se han reportado similares verificaciones en estudiantes de la costa norte, la selva o el sur del país. En estas regiones, los estudiantes tienen características culturales y académicas muy diferenciadas y pueden mostrar disimilitudes importantes en sus hábitos de estudio y en las dimensiones que los componen (Reynaga, 2009).

El problema de la poca representatividad nacional de la evidencia psicométrica sobre el CASM-85 tiene potenciales repercusiones en dos niveles, uno descriptivo y otro comparativo. Con nivel descriptivo nos referimos a cuánto pueden verse afectadas las interpretaciones que los educadores/psicólogos de cada ciudad realizan al utilizar este instrumento en sus respectivas localidades, mientras están usando la referencia limeña para hacer tales interpretaciones (norma de calificación del manual). En la práctica, aunque una fracción importante de usuarios se concentra en la capital (Beteta, 2008; Chilca, 2017; Cuadros, Morán, \& Torres, 2017; Huamán, 2017; Lucas, 2017; Rosales, 2016), existen otros estudios que evidencian el uso del CASM-85 en distintas ciudades del interior de Perú como Arequipa, Trujillo, Juliaca e Iquitos (Huayta \& Huayllani, 2014; Atamari, 2014; Meza, 2016; Miranda, 2017; Pérez \& Silva, 2018; Salas, 2017). Los autores de estos estudios en el interior del país confían en las propiedades psicométricas reportadas para población limeña, y en su norma de calificación, sin que haya certeza de que el instrumento funciona idénticamente bien para estudiantes de cualquiera de estas ciudades. Más aún, y a nivel comparativo, actualmente no se cuenta con evaluaciones de la invarianza de medida entre ciudades que permitan garantizar que el mismo constructo postulado en Lima se está midiendo idénticamente a lo largo del país o sustentar el uso de una norma de calificación única (Meredith, 1993). 
De hecho, creemos que algunas dimensiones del CASM-85 podrían verse afectadas por el sexo, la edad y la ciudad de residencia. Por ejemplo, en ciertas zonas rurales las mujeres desde una edad temprana, y a diferencia de los hombres, tienen más funciones en el hogar asignadas por los padres, lo que reduce su tiempo para estudiar y realizar sus tareas académicas diariamente (Cueto, 2004; Reynaga, 2009; Vicuña, 2005). La edad es otro factor que puede alterar los hábitos de estudio, especialmente en la adolescencia tardía cuando otras actividades sociales comienzan a ganar más importancia frente al estudio. Por otra parte, en las regiones más pobres de la sierra y selva peruana, los estudiantes tienen menos acceso a luz eléctrica o servicios educativos, lo que modifica los horarios o la frecuencia con la que pueden estudiar; o por el contrario, tienen menos acceso a distractores tecnológicos como smartphones, videojuegos, equipos de sonido, televisores y similares (Instituto Nacional de Estadística e Informática, 2015). Este contraste en el acceso a distractores no es solo posible entre regiones, sino también entre la ciudad y el campo o entre colegios públicos o privados; es decir, entre toda aquella diferencia ligada a una de tipo socioeconómico. Por ejemplo, vivir en hogares estrechos o superpoblados incrementa la probabilidad de sufrir constantes desconcentraciones al momento de estudiar debido a las inevitables interrupciones de algún familiar (Instituto Nacional de Estadística e Informática, 2015, 2018; Vicuña, 2005). En suma, los hábitos de estudio pueden variar estructuralmente según el sexo, la edad, la ciudad o el tipo de colegio del estudiante, afectando potencialmente la invarianza de su medición con instrumentos como el CASM-85.

Nuestro objetivo general fue revisar las propiedades psicométricas del CASM-85 en estudiantes de secundaria de cuatro ciudades del norte, sur y centro del Perú. Dicho objetivo general se divide en los siguientes objetivos específicos: 1) confirmar la estructura de cinco dimensiones del CASM-85 en dichas poblaciones; 2) evaluar su invarianza de medida a través de subgrupos según edad, sexo, colegio y ciudad; 3) estimar la confiabilidad del CASM-85 y 4) evaluar la validez de criterio del CASM-85 respecto al rendimiento académico.

\section{Método}

\section{Diseño}

El estudio es transversal de tipo psicométrico en el que nos ocupamos de revisar la estructura de cinco dimensiones, invarianza de medida, validez de criterio y confiabilidad del CASM-85. Para el análisis de invarianza, las variables dependientes fueron los ítems del CASM-85 y las covariables fueron el sexo, la edad, el colegio y la ciudad. Para la validez de criterio, la variable dependiente fue el rendimiento académico y la independiente, los hábitos de estudio (CASM-85). La estructura de cinco dimensiones fue revisada mediante análisis factorial y la confiabilidad mediante consistencia interna.

\section{Participantes}

Los participantes del estudio fueron adolescentes de ambos sexos con edades entre los 11 y 18 años, estudiantes del primero al quinto año de educación secundaria regular de colegios particulares y nacionales de cuatro ciudades peruanas: Arequipa (sierra sur), Chimbote (costa norte), Lima (costa centro) y Pucallpa (selva norte). Estas ciudades fueron elegidas intencionalmente dado que representan a regiones peruanas cuya población tiene características culturales claramente diferenciadas. En cada ciudad, dos colegios fueron seleccionados para representar a sus similares públicos y privados. Luego, se seleccionaron en forma aleatoria a los estudiantes a ser evaluados en cada colegio, que en conjunto debían sumar e» 400 alumnos por ciudad. Así, el CASM-85 pudo aplicarse a 760 estudiantes de Arequipa, 504 de Chimbote, 404 de Lima y 407 de Pucallpa. Solo para una submuestra (692 en Arequipa y 401 en Pucallpa), se tuvo acceso a los datos de rendimiento académico de los estudiantes. Las características de la muestra general son visibles en la Tabla 1 . 
Tabla 1

Características de las poblaciones evaluadas $(N=2075)$

\begin{tabular}{|c|c|c|c|c|c|c|c|c|}
\hline & \multirow{2}{*}{\multicolumn{2}{|c|}{$\frac{\mathrm{A}}{(n=760)}$}} & \multirow{2}{*}{\multicolumn{2}{|c|}{$\begin{array}{c}\text { Ch } \\
(n=504)\end{array}$}} & \multirow{2}{*}{\multicolumn{2}{|c|}{$\frac{\mathrm{L}}{(n=404)}$}} & \multirow{2}{*}{\multicolumn{2}{|c|}{$\begin{array}{c}\mathrm{P} \\
(n=407)\end{array}$}} \\
\hline & & & & & & & & \\
\hline & $n$ & $(\%)$ & $n$ & $(\%)$ & $n$ & $(\%)$ & $n$ & $(\%)$ \\
\hline \multicolumn{9}{|l|}{ Colegio } \\
\hline Nacional & 155 & 20.4 & 246 & 48.8 & 228 & 56.4 & 237 & 58.2 \\
\hline Particular & 605 & 79.6 & 258 & 51.2 & 176 & 43.6 & 170 & 41.8 \\
\hline \multicolumn{9}{|l|}{ Año de secundaria } \\
\hline Primero & 179 & 23.6 & 114 & 22.6 & 84 & 20.8 & 78 & 19.2 \\
\hline Segundo & 178 & 23.4 & 104 & 20.6 & 80 & 19.8 & 77 & 18.9 \\
\hline Tercero & 129 & 17.0 & 94 & 18.7 & 81 & 20.1 & 91 & 22.4 \\
\hline Cuarto & 150 & 19.7 & 97 & 19.3 & 80 & 19.8 & 80 & 19.7 \\
\hline Quinto & 124 & 16.3 & 95 & 18.9 & 79 & 19.6 & 81 & 19.9 \\
\hline \multicolumn{9}{|l|}{ Sexo } \\
\hline Femenino & 455 & 59.9 & 297 & 58.9 & 211 & 52.3 & 192 & 47.2 \\
\hline Masculino & 305 & 40.1 & 207 & 41.1 & 193 & 47.8 & 215 & 52.8 \\
\hline \multicolumn{9}{|l|}{ Edad (años) } \\
\hline media (desviación estándar) & 13.8 & $(1.6)$ & 14.4 & (1.6) & 14.2 & $(1.5)$ & 14.4 & $(1.5)$ \\
\hline
\end{tabular}

Nota: $\mathrm{A}=$ Arequipa $; \mathrm{CH}=$ Chimbote $; \mathrm{L}=$ Lima; $\mathrm{P}=$ Pucallpa

\section{Instrumento}

El Inventario de Hábitos de Estudio CASM-85 es el instrumento y objeto del estudio. Su versión original data de 1985, con una revisión de 1998 que fue reimpresa en el 2005 y 2014. La reimpresión del 2014 sugirió la adición de dos ítems, pero no presentó ninguna evidencia de su proceso de validación, razón por la cual decidimos usar la escala intacta de 53 ítems de 1998 y 2005. Los ítems están distribuidos en cinco áreas o dimensiones: 1) ¿Cómo estudia Ud.? (12 ítems), 2) ¿Cómo hace sus tareas? (10 ítems), 3) ¿Cómo prepara sus exámenes? (11 ítems), 4) ¿Cómo escucha las clases? (12 ítems) y 5) ¿Qué acompaña sus momentos de estudio? (8 ítems). Estas dimensiones refieren a la categorización de conductas observables comunes en la población objetivo, así identificadas por el autor, por lo que tales dimensiones no señalan la descripción de un método de estudio en particular (Vicuña, 2014). El CASM-85 tiene 35 ítems inversos, distribuidos aleatoriamente entre y dentro de cada una de las cinco subescalas del inventario. De esa forma, sabemos no refuerzan artificialmente la dimensionalidad del CASM-85. Los ítems del CASM-85 son de respuesta dicotómica (siempre-nunca) y se califican con 1 para los hábitos positivos y 0 para los negativos; es decir, a mayor puntaje, mejores hábitos, tanto para las subescalas (dimensiones) como para la escala global. Los detalles sobre cómo se clasifican los hábitos entre positivos y negativos pueden revisarse en el manual del instrumento (ver criterio de calificación); no obstante, en el CASM-85, los hábitos positivos son aquellos asociados al buen rendimiento académico, independientemente del coeficiente intelectual del estudiante (Vicuña, 2014). El puntaje crudo (e.g., 053 para la escala global) se transforma en puntajes $T$ (20 a 80) y se interpreta en 6 niveles o categorías: muy positivo, positivo, tendencia positiva, tendencia negativa, negativa y muy negativa. La aplicación típica del CAMS-85 dura entre 15 y 20 minutos. La versión original del CASM-85 reporta una buena confiabilidad ( $>.90$ en consistencia interna y test-retest) y validez 
de criterio con el rendimiento académico (Vicuña, 1985, 1998, 2005, 2014).

\section{Variables}

Las covariables del análisis de invarianza fueron el sexo (femenino/masculino), la edad (en años), el colegio (particular o nacional) y la ciudad (Arequipa, Chimbote, Lima, Pucallpa). El rendimiento académico (promedio general del estudiante) fue la variable dependiente al evaluar la validez de criterio.

\section{Procedimiento}

El CASM-85 fue aplicado en uno de dos formatos, virtual o físico. El formato físico fue aplicado en los salones de clase, en horario regular y por personal entrenado. En aquellos colegios que disponían de laboratorios de cómputo, se aplicó la versión virtual utilizando la plataforma gratuita LimeSurvey. La aplicación virtual también fue asistida por personal entrenado. En todos los casos, se procuró que las condiciones de aplicación fueran similares y no afectaran las evaluaciones.

\section{Análisis de datos}

El análisis se inició con la reorientación de ítems inversos, la evaluación de su distribución, así como el cálculo de la correlación ítem-ítem de cada dimensión del CASM-85 por separado (matrices tetracóricas) y de la correlación ítem-resto, también por cada dimensión. A partir de dicha exploración se detectaron 8 ítems muy heterogéneos respecto a sus propias dimensiones, los mismos cuyo contenido fue evaluado por el equipo de investigadores. De dicha evaluación se concluyó que su remoción no atentaba contra la validez de contenido de cada dimensión del instrumento (representatividad de dominio). Así, se decidió realizar todos los análisis subsiguientes con dos formas del CASM-85, la original (53 ítems) y la reducida (45 ítems), para brindar a los lectores una fácil comparación y elección de uso. Para ambas formas se evaluó el modelo de cinco dimensiones propuesto por Vicuña $(1985,1998,2005)$ mediante un análisis factorial confirmatorio (AFC). En dicho análisis, el método de Mínimos Cuadrados Ponderados Robustos (WLSMV por sus siglas en inglés) fue aplicado para la estimación de los parámetros del modelo de medida, toda vez que este método es apropiado para ítems categóricos con distribución latente no-normal (Beauducel \& Herzberg, 2006; Cheng-Hsien, 2016; DiStefano \& Morgan, 2014).

Para valorar el ajuste de estos modelos de medida, hemos reportado los siguientes índices: Comparative Fit Index (CFI), Tucker-Lewis Index (TLI), Standardized Root Mean Squared Residual (SRMR) y Root Mean Squared Error of Approximation (RMSEA), toda vez que el tradicional test de Chicuadrado es muy sensible al tamaño muestral grande (Brown, 2014). Para las interpretaciones, CFI y TLI representan un ajuste aceptable cuando sus valores son $\geq .90$ y buen ajuste cuando son $\geq .95$. Por otro lado, se espera que SRMR y RMSEA sean $\leq .08$ cuando haya un buen ajuste del modelo de medida (Beauducel \& Wittmann, 2005; Hu \& Bentler, 1999). Este AFC fue realizado para cada ciudad por separado y se reporta en la Tabla 2 para ambas formas del CASM-85 (original y reducida).

Para evaluar la invarianza de medida se utilizaron modelos MIMIC (Multiple Indicator, Multiple Cause), evaluando la invarianza de los interceptos de los indicadores y las diferencias de medias de las dimensiones latentes, todo a través de grupos según edad, sexo, colegio y ciudad (covariables), y teniendo en cuenta la misma estructura de 5 dimensiones evaluada previamente en el AFC. El método MIMIC ha sido preferido al AFC multigrupos, toda vez que la muestra recolectada no es lo suficientemente grande como para garantizar estimaciones robustas mediante el modelado multigrupos (Brown, 2014). El modelado MIMIC analiza cada covariable por separado, donde para cada una se estiman y comparan dos modelos: 1) un modelo MIMIC saturado donde la covariable explica a todos los ítems observados, pero no a las dimensiones latentes y 2) un modelo de intercepto invariante donde la covariable explica a todas las 
Tabla 2

Índices de ajuste del modelo de cinco dimensiones del CASM-85 en cada población evaluada $(N=2075)$

\begin{tabular}{|c|c|c|c|c|c|}
\hline CASM-85 & Índices & $\begin{array}{c}\text { Arequipa } \\
(n=760)\end{array}$ & $\begin{array}{l}\text { Chimbote } \\
(n=504)\end{array}$ & $\begin{array}{c}\text { Lima } \\
(n=404)\end{array}$ & $\begin{array}{l}\text { Pucallpa } \\
(n=407)\end{array}$ \\
\hline \multirow{5}{*}{$\begin{array}{c}\text { Forma } \\
\text { original } \\
\text { (53 ítems) }\end{array}$} & $\chi^{2}(\mathrm{gl})$ & $2398(1315)$ & $2170(1315)$ & $2020(1315)$ & 1955 (1315) \\
\hline & CFI & .879 & .827 & .839 & .838 \\
\hline & TLI & .874 & .819 & .831 & .830 \\
\hline & SRMR & .079 & .094 & .102 & .103 \\
\hline & RMSEA & .033 & .036 & .036 & .035 \\
\hline \multirow{5}{*}{$\begin{array}{c}\text { Forma } \\
\text { reducida } \\
\text { (45 ítems) }\end{array}$} & $\chi^{2}(\mathrm{gl})$ & $1568(935)$ & $1512(935)$ & $1332(935)$ & $1200(935)$ \\
\hline & CFI & .923 & .873 & .907 & .930 \\
\hline & TLI & .919 & .865 & .902 & .925 \\
\hline & SRMR & .071 & .088 & .091 & .088 \\
\hline & RMSEA & .030 & .035 & .032 & .026 \\
\hline
\end{tabular}

Nota: $\chi^{2}(\mathrm{gl})=$ Chi-cuadrado (grados de libertad); CFI = Comparative Fit Index; TLI = Tucker-Lewis Index; SRMR = Standardized Root Mean Squared Residual; RMSEA = Root Mean Squared Error of Approximation.

dimensiones latentes, pero no a los ítems. Para estos modelos, las variables sexo (hombres $=0$, mujeres $=$ 1) y colegio (particular $=0$, nacional $=1$ ) fueron dicotómicas, la edad continua y las ciudades recodificadas como variables dummy, donde Arequipa fue el grupo de referencia. Cuando el ajuste del modelo de intercepto invariante no se reduce en forma apreciable respecto al modelo saturado (e.g., si $\Delta \mathrm{CFI}$, $\Delta \mathrm{TLI}, \Delta \mathrm{RMSEA}$ son $\leq .01$ ), se concluye que las diferencias entre grupos (según covariables) son enteramente atribuibles a diferencias en las dimensiones latentes y no a problemas sistemáticos con el CASM-85 (e.g., funcionamiento diferencial de los ítems) (Marsh, Morin, Parker, \& Kaur, 2014).

Los índices de ajuste de los modelos MIMIC se reportan para ambas formas del CASM-85 (original y reducida) en la Tabla 3. Las diferencias de dimensiones latentes se reportan en la Tabla 4 para justificar o no el eventual uso de una norma de calificación única, tras mostrar betas no estandarizados (con error estándar y p-valor) entre cada covariable y cada dimensión. La confiabilidad se ha estimado con los coeficientes Alpha y Omega para la muestra global y en cada ciudad. Para el Alpha, hemos utilizado la fórmula proporcionada por Cronbach (1951) y calculada a partir de correlaciones tetracóricas, mientras que para el Omega hemos utilizado la fórmula de Bollen (1980) y Raykov (2001). Para ambos coeficientes, valores $\geq .90$ son recomendados para evaluaciones individuales, mientras que valores $\geq .70$ son aceptables para evaluaciones grupales (Aiken, 1985). La validez de criterio entre el CASM85 y el rendimiento académico fue revisada de dos maneras: 1) una $r$ de Pearson entre el promedio general de los alumnos y el puntaje global crudo del CASM-85, y 2) un modelo de regresión lineal múltiple donde el promedio general era la variable dependiente y los puntajes de las subescalas (dimensiones) del CASM-85 eran los predictores. Valores positivos de la $r$ de Pearson y de las betas de los modelos de regresión se interpretan como asociación directa; es decir, a mayor puntaje del CASM-85 mayor rendimiento académico. El factor de inflación de la varianza (VIF) fue calculado para los modelos de regresión, de modo que valores $<10$ indicarían ausencia de multicolinealidad. Todos los análisis estadísticos se realizaron con Stata/IC v15 (StataCorp, 2017) y los paquetes R lavaan y semTools (Jorgensen, Pornprasertmanit, Schoemann, \& Rosseel, 2018), considerando valores $p<.05$ como estadísticamente significativos. 


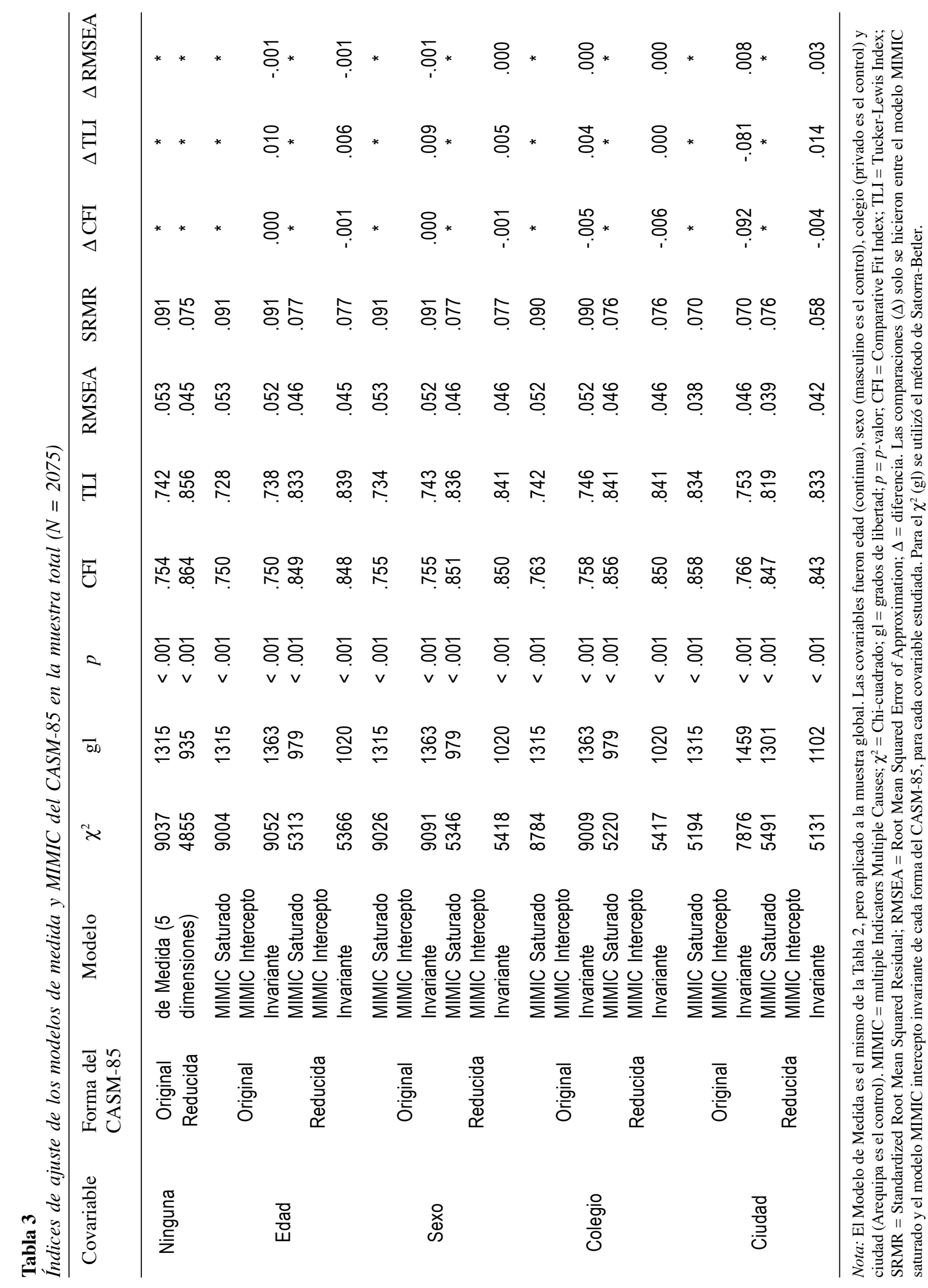


Tabla 4

Diferencia de medias de dimensiones latentes del CASM-85 entre grupos según edad, sexo, colegio y ciudad ( $N=2075)$

\begin{tabular}{|c|c|c|c|c|c|c|c|}
\hline \multirow{2}{*}{ Covariable } & \multirow{2}{*}{$\begin{array}{l}\text { Forma del } \\
\text { CASM-85 }\end{array}$} & \multirow{2}{*}{ Modelo } & \multicolumn{5}{|c|}{ Dimensiones del CASM-85 } \\
\hline & & & I & II & III & IV & $\mathrm{V}$ \\
\hline \multirow{6}{*}{ Edad } & \multirow{3}{*}{ Original } & Diferencia de medias & -.027 & .010 & -.016 & -.023 & .011 \\
\hline & & Error estándar & .009 & .003 & .004 & .005 & .005 \\
\hline & & $p$-valor & .005 & .004 & $<.001$ & $<.001$ & .019 \\
\hline & \multirow{3}{*}{ Reducida } & Diferencia de medias & -.026 & .011 & -.014 & -.022 & .011 \\
\hline & & Error estándar & .010 & .003 & .004 & .005 & .005 \\
\hline & & $p$-valor & .006 & .002 & $<.001$ & $<.001$ & .019 \\
\hline \multirow{6}{*}{ Sexo } & \multirow{3}{*}{ Original } & Diferencia de medias & .095 & -.001 & .018 & .002 & .001 \\
\hline & & Error estándar & .030 & .005 & .009 & .014 & .014 \\
\hline & & $p$-valor & .002 & .801 & .039 & .907 & .069 \\
\hline & \multirow{3}{*}{ Reducida } & Diferencia de medias & .098 & -.001 & .016 & .001 & .001 \\
\hline & & Error estándar & .031 & .006 & .008 & .014 & .014 \\
\hline & & $p$-valor & .001 & .795 & .044 & .932 & .943 \\
\hline \multirow{6}{*}{ Colegio } & \multirow{3}{*}{ Original } & Diferencia de medias & .053 & .004 & -.013 & .007 & .007 \\
\hline & & Error estándar & .030 & .005 & .008 & .014 & .014 \\
\hline & & $p$-valor & .076 & .487 & .122 & .633 & .598 \\
\hline & \multirow{3}{*}{ Reducida } & Diferencia (Dif.) de medias & .048 & .005 & -.007 & .004 & .007 \\
\hline & & Error estándar & .030 & .006 & .007 & .014 & .014 \\
\hline & & $p$-valor & .112 & .369 & .315 & .785 & .595 \\
\hline \multirow{18}{*}{ Ciudad } & \multirow{9}{*}{ Original } & CHIMBOTE: Dif. de medias & .371 & .223 & -.115 & -.208 & .260 \\
\hline & & CHIMBOTE: Error estándar & .047 & .029 & .023 & .028 & .035 \\
\hline & & CHIMBOTE: $p$-valor & $<.001$ & $<.001$ & $<.001$ & $<.001$ & $<.001$ \\
\hline & & LIMA: Dif. de medias & .047 & .040 & -.003 & .025 & .079 \\
\hline & & LIMA: Error estándar & .043 & .025 & .013 & .019 & .031 \\
\hline & & LIMA: $p$-valor & .280 & .102 & .828 & .193 & .011 \\
\hline & & PUCALLPA: Dif. de medias & .241 & -.015 & .015 & .070 & -.015 \\
\hline & & PUCALLPA: Error estándar & .047 & .024 & .013 & .021 & .030 \\
\hline & & PUCALLPA: $p$-valor & $<.001$ & .543 & .250 & .001 & .625 \\
\hline & \multirow{9}{*}{ Reducida } & CHIMBOTE: Dif. de medias & -.003 & .234 & -.103 & -.204 & .259 \\
\hline & & CHIMBOTE: Error estándar & .053 & .030 & .024 & .028 & .035 \\
\hline & & CHIMBOTE: $p$-valor & .952 & $<.001$ & $<.001$ & $<.001$ & $<.001$ \\
\hline & & LIMA: Dif. de medias & -.036 & .044 & -.003 & .023 & .078 \\
\hline & & LIMA: Error estándar & .057 & .024 & .011 & .018 & .031 \\
\hline & & LIMA: $p$-valor & .533 & .064 & .762 & .216 & .010 \\
\hline & & PUCALLPA: Dif. de medias & .278 & -.004 & .012 & .064 & -.014 \\
\hline & & PUCALLPA: Error estándar & .061 & .023 & .011 & .020 & .030 \\
\hline & & PUCALLPA: $p$-valor & .000 & .846 & .279 & .001 & .650 \\
\hline
\end{tabular}

Nota: Las diferencias de medias se han obtenido del modelo de intercepto invariante. Las covariables fueron edad (continua), sexo (masculino es el control), colegio (privado es el control) y ciudad (Arequipa es el control). 


\section{Resultados}

Las características de los estudiantes evaluados en las cuatro ciudades son visibles en la Tabla 1. La distribución entre alumnos y alumnas es aproximadamente 2:3 en Arequipa y Chimbote, y 1:1 en Lima y Pucallpa. Los alumnos de Arequipa provienen en su mayoría de colegios particulares, mientras que en Pucallpa lo hacen de nacionales. La distribución en años de secundaria es bastante homogénea en todas las ciudades, mientras que la edad promedio está alrededor de 14 años en todos los grupos.

La estructura de cinco dimensiones del CASM-85 presenta mejores indicadores de bondad de ajuste para la forma reducida (45 ítems) que para la forma original (53 ítems) (Tabla 2). Los ítems heterogéneos que se retiraron para obtener la forma reducida fueron los ítems $12,14,22,23,27,31,36$ y 37 de la forma original (Vicuña, 2005). Para la forma original, el CFI y TLI es $<.9$ para los alumnos de todas las ciudades, mientras que para la forma reducida es $<.9$ solo en Chimbote. El SRMR y el RMSEA son menores para la forma reducida en todas las ciudades, aunque solo para Arequipa los valores de SRMR son $<.08$.

Los modelos MIMIC saturado y de intercepto invariante son semejantes para todas las variables evaluadas, con excepción de la variable ciudad para la forma original del CASM-85 (Tabla 3). Para la variable edad, los valores absolutos de $\Delta \mathrm{CFI}, \Delta \mathrm{TLI}$, y $\triangle$ RMSEA son $\leq .01$. Para las variables sexo y colegio, estos mismos valores absolutos son $<.01$. Para edad, sexo y colegio, los valores CFI y TLI son $<.8$ para la forma original del CASM-85 y $>.8$ para su forma reducida. Para todas las variables estudiadas, el RMSEA es siempre < .06 y el SRMR es siempre $<.10$. Solo para la variable ciudad se encontró una diferencia notable entre los modelos MIMIC saturado $\mathrm{y}$ de intercepto invariante $(|\Delta \mathrm{CFI}|=.09,|\Delta \mathrm{TLI}|=.08)$, que alerta sobre falta de invarianza de medida de la forma original del CASM-85 a través de las cuatro ciudades. Las diferencias entre las medias de las dimensiones latentes son visibles en la Tabla 4 para ambas versiones del CASM-85. Para la edad, todas las dimensiones del CASM-85 presentan diferencias significativas $(p<.05)$; sin embargo, la diferencia absoluta por cada año nunca es mayor a .27 puntos. Para el sexo, algunas diferencias son significativas, pero cuando lo son la diferencia absoluta nunca es mayor a .10 puntos. Para el colegio, ninguna diferencia fue significativa. Para las ciudades, algunas diferencias lo fueron, pero su valor absoluto nunca fue mayor a .28 puntos.

Respecto a la confiabilidad, todos los valores calculados de Alpha y Omega son $\geq .7$ para las formas original y reducida del CASM-85 (Tabla 5). De hecho, para todas las ciudades con excepción de Chimbote, la confiabilidad de la forma reducida mostró ser mayor que la de la forma original. Aproximando al primer decimal, la confiabilidad de la forma reducida fue $\geq$ .8 para los alumnos de todas las ciudades. En la misma tabla, se aprecia que la media global del CASM-85 es más alta en Chimbote $\left(\bar{x}_{f-\text { orig }}=28.6 \pm\right.$ $8.1 ; \bar{x}_{f-\text { redu }}=24.5 \pm 7.2$ ) que en las otras ciudades $\left(\bar{x}_{f-\text { orig }} \approx 25 \pm 7 ; \bar{x}_{f-\text { redu }} \approx 20 \pm 7\right.$ ), para ambas formas original y reducida.

La relación entre el rendimiento académico y el CASM-85 fue positiva y significativa, pero de fuerza débil. Los promedios de calificación disponibles de 1092 alumnos fueron incluidos en este análisis, encontrándose una correlación de $r=.17(p<.001)$ entre estos promedios y el puntaje global crudo del CASM-85 para su forma original, y una $r=.18(p<$ .001) para su forma reducida. Para la forma original, el modelo de regresión lineal que tenía como predictores a cada una de las dimensiones del CASM85 y como variable dependiente al rendimiento académico $(\mathrm{F}(5,1086)=10, p<.001)$, mostró un $\mathrm{R}^{2}$ ajustado de .04 donde solo la segunda dimensión del CASM-85 tuvo una relación significativa $(\beta=.10$, $p<.01)$ con el rendimiento académico, verificándose un VIF general de 1.45. Para la forma reducida del CASM-85, un modelo de regresión similar (F $(5,1086)$ 
Tabla 5

Confiabilidad, media y desviación estándar del CASM-85, global y por cada población evaluada

\begin{tabular}{ccccccc}
\hline $\begin{array}{c}\text { Forma del } \\
\text { CASM-85 }\end{array}$ & Índice & $\begin{array}{c}\text { Global } \\
(N=20175)\end{array}$ & $\begin{array}{c}\text { Arequipa } \\
(n=760)\end{array}$ & $\begin{array}{c}\text { Chimbote } \\
(n=504)\end{array}$ & $\begin{array}{c}\text { Lima } \\
(n=404)\end{array}$ & $\begin{array}{c}\text { Pucallpa } \\
(n=407)\end{array}$ \\
\hline \multirow{2}{*}{ Original } & Alpha & .84 & .81 & .89 & .86 & .85 \\
$(53$ ítems $)$ & Omega & .75 & .68 & .85 & .74 & .73 \\
& media \pm de & $26.2 \pm 7.2$ & $25.2 \pm 6.3$ & $28.6 \pm 8.1$ & $25.7 \pm 7.1$ & $25.6 \pm 6.9$ \\
\hline \multirow{2}{*}{$\begin{array}{c}\text { Reducida } \\
(45 \text { ítems })\end{array}$} & Alpha & .86 & .84 & .89 & .88 & .87 \\
& Omega & .81 & .77 & .84 & .81 & .81 \\
& media \pm de & $21.5 \pm 6.9$ & $20.5 \pm 6.3$ & $24.5 \pm 7.2$ & $20.8 \pm 6.9$ & $20.4 \pm 6.6$ \\
\hline
\end{tabular}

Nota $:$ media \pm de $=$ media \pm desviación estándar.

$=11, p<.001)$, mostró un $\mathrm{R}^{2}$ ajustado de .04 donde la segunda dimensión $(\beta=.08, p=.01)$ y la tercera dimensión $(\beta=.07, p=.03)$ del CASM-85 mostraron una relación significativa con el rendimiento académico, verificándose un VIF general de 1.64.

Otros resultados descriptivos o productos intermedios del análisis psicométrico, como las tablas de distribución de los ítems o matrices de correlación, pueden ser solicitados al autor corresponsal vía correo electrónico.

\section{Discusión}

Tras el análisis de las propiedades psicométricas del Inventario de Hábitos de Estudio CASM-85 en estudiantes peruanos de secundaria, los resultados mostraron que la estructura de cinco dimensiones tuvo mejor bondad de ajuste para la forma reducida del CASM-85 que para su forma original. Con respecto a la invarianza de medida, ambas formas del CASM85 mostraron ser equivalente a través de los grupos según edad, sexo y tipo de colegio (nacional/particular); no obstante, solo la forma reducida mostró equivalencia a través de las poblaciones de las cuatro ciudades (Arequipa, Chimbote, Lima y Pucallpa). Las diferencias de medias latentes entre grupos según edad, sexo, tipo de colegio y ciudad nunca fueron mayores a .28 puntos para todas las dimensiones del
CASM-85. La confiabilidad del CASM-85 en su forma reducida fue siempre mejor o igual que la de su forma original, en estudiantes de todas las ciudades con excepción de Chimbote. Se confirmó una moderada validez de criterio para ambas formas, toda vez que la relación entre el rendimiento académico y el CASM-85 fue positiva y significativa, aunque de fuerza débil.

Hemos confirmado que el CASM-85 tiene una estructura de cinco dimensiones, especialmente clara en su forma reducida. Aunque dicha forma reducida mostró mejor ajuste que la forma original, tal ajuste alcanzó un nivel aceptable (CFI y TLI > .90) solo en tres de las cuatro ciudades donde se hizo el estudio. No obstante, nuestra posición es que estos resultados todavía avalan la propuesta de cinco dimensiones realizada originalmente por el autor (Vicuña, 1985, 1998,2005 , 2014). Para la forma reducida, se puede concluir que la estructura de cinco dimensiones sigue siendo funcional si se considera que el método empleado (WLSMV) es particularmente riguroso y que los indicadores de bondad de ajuste reportados en Chimbote no están muy alejados de los límites aceptables (e.g., que el CFI y TLI aproximado a un decimal es .9 y recordando que su RMSEA es $<.08$ ). Para la forma original del CASM-85, nuestros resultados son más conservadores (CFI y TLI < .90) que otros reportados previamente por Armas (2017) 
(GFI, RFI y NFI > .90), quien de hecho aplicó un método de estimación diferente al nuestro (Mínimos Cuadrados No Ponderados o ULS por sus siglas en inglés, frente a nuestro WLSMV). Actualmente, WLSMV es el método más apropiado y riguroso para análisis factorial confirmatorio de instrumentos con mediciones no-continuas que tienen distribución latente no-normal (Beauducel \& Herzberg, 2006; ChengHsien, 2016; DiStefano \& Morgan, 2014) como es el caso del CASM-85 y, mientras no presente problemas de convergencia, lo preferimos frente a opciones no ponderadas como ULS o ULS robusto. De hecho, en un estudio semejante, Rebaza (2016) también reportó indicadores de bondad de ajuste más conservadores que los reportados por Armas (2017) tras haber estudiado a una población cercana y culturalmente similar (Huamachuco y Otuzco son capitales de provincias vecinas de la sierra de La Libertad). Aquí nuevamente las diferencias de ajuste parecen provenir del método de estimación, pues para estudiar la forma original del CASM-85, Rebaza (2016) utilizó el método de Máxima Verosimilitud $(\mathrm{ML})$, reportando CFI y GFI < .90. En nuestra experiencia, cuando los tres métodos convergen, los resultados de ML y WLSMV suelen ser más conservadores que los de ULS. Sin embargo, actualmente no se cuenta con estudios de simulación minuciosos que permitan realizar una comparación más formal para nuestro escenario particular de análisis (cinco dimensiones, ítems dicotómicos, latentes no-normales, etc.). Dicho esto, concluimos que la evidencia psicométrica acumulada se inclina en favor de un mejor ajuste del modelo de cinco dimensiones para la forma reducida del CASM-85.

La forma original del CASM-85 no muestra señales de invarianza de medida entre estudiantes de diferentes ciudades del país. La implicancia práctica de este hallazgo es que el usuario no puede estar seguro de que con esta forma del CASM-85 se puede medir el mismo constructo en cada una de estas poblaciones. Eso limita seriamente las comparaciones entre grupos de estudiantes según ciudades y complejiza el uso de una norma única a nivel nacional; la cual, de hecho, se suele usar para interpretar los resultados de las mediciones realizadas con este instrumento en diferentes lugares del país. Sin embargo, tras retirarse los ítems más heterogéneos, se obtiene una versión reducida del CASM-85 que sí muestra invarianza de medida a través de las ciudades evaluadas. Al revisar el contenido de algunos de los ítems retirados, es posible entender la naturaleza y fuente de la distorsión que impide la invarianza. Por ejemplo, el ítem 14 pregunta al estudiante si al hacer las tareas este pone en práctica el hábito de leer la pregunta, buscar en el libro, leer todo y posteriormente contestar según cómo el estudiante haya comprendido. Aquí, la fuente de varianza entre ciudades puede venir del hecho de que no en todas ellas el estilo de tareas que los docentes dejan a sus alumnos es necesariamente el mismo (e.g., preguntas y respuestas), o que el hacer las tareas requiera del uso de libros con la misma frecuencia o con el mismo acceso a ellos (e.g., Internet o textos no escolares). En otro ejemplo, los ítems 22 y 31 preguntan sobre el hábito de «empezar con lo más difícil y terminar con lo más fácil» cuando se hacen tareas o se estudia para los exámenes, asumiendo que esta práctica administraría mejor los efectos de la fatiga. No obstante, no hay una garantía de que en todas las ciudades las tareas escolares o los temas para los exámenes sean igual de largos (facilitando la clasificación y ordenamiento sugeridos), o que las tareas contengan preguntas con notables diferencias en su grado de dificultad la mayoría de las veces que se las asignan. Estos ítems, dependen mucho de prácticas escolares que pueden variar significativamente de ciudad en ciudad, mientras que la mayoría de los otros ítems del CASM-85 sí están ligados a prácticas escolares mucho más estables. Por otro lado, la forma reducida del CASM-85 ha mostrado diferencias de medias latentes muy pequeñas ( $<.29$ puntos), considerando que las dimensiones del CASM-85 están escaladas desde 0 hasta un máximo de 12 puntos. Así, la elaboración de normas estratificadas por edad, sexo o ciudad no tienen una justificación práctica, validando el uso de una norma única a nivel nacional. En suma, solo la forma 
reducida del CASM-85 brinda una medida equivalente y suficientemente estable a través de grupos de estudiantes clasificados según sexo, edad, tipo de colegio y, particularmente, entre grupos de distintas ciudades del país.

La confiabilidad del CASM-85 es buena para evaluaciones grupales, y regular para evaluaciones individuales. En todas las ciudades, la consistencia interna fue prácticamente $\geq .7$, tanto para la forma reducida como para la original del CASM-85. Sin embargo, si consideramos el criterio de $\geq .9$ para confiar en evaluaciones individuales, solo la forma reducida se aproxima más a este valor, razón por la que concluimos solo una confiabilidad regular para este tipo de evaluaciones. Por el contrario, los resultados mostrados por Vicuña (1998) en su revisión de la forma original reportan un valor de .9 más optimista. No obstante, es preciso acotar que en dicho estudio se empleó un método de estimación diferente al nuestro al aplicar el método Kuder-Richardson. Sin embargo, en Otuzco la evaluación con estudiantes de secundaria reportó valores más discretos usando el mismo índice $(\mathrm{KR}-20>$.85) (Rebaza, 2016). Finalmente, el estudio en Huamachuco utilizó un índice similar al nuestro, el coeficiente de Omega, concluyendo valores de confiabilidad $\geq .7$ similares a los nuestros (Armas, 2017). Desde que el Omega es un índice específicamente formulado para escalas multidimensionales como el CASM-85, preferimos su estimación más conservadora la cual aún reporta una buena consistencia interna para cualquier evaluación grupal que el usuario desee realizar. En términos prácticos, esto significa que el psicólogo/educador puede aplicar el instrumento en su salón de clase o en una investigación y las estimaciones de los hábitos de estudio para el grupo como un todo serán muy confiables.

Se confirmó la validez de criterio del CASM-85 con el rendimiento académico, aunque la asociación entre ellos fue débil, probablemente porque el rendimiento académico tiene causas multifactoriales. De hecho, originalmente el mismo autor del CASM-
85 (Vicuña, 1998) exploró la diferencia entre el rendimiento académico de estudiantes con hábitos positivos $(M=14.16, D E=1.54, n=33)$ y de aquellos con hábitos negativos $(M=12.32, D E=1.11, n=$ $58)$, encontrando también un tamaño de efecto débil $\left(\Delta_{\text {diferencia de medias }}=1.85, \mathrm{CI} 95 \%=1.2-2.5\right.$ para una escala de rendimiento académico $0-20, t(6.1)=51.2$, $p<.001)$. Nuestro estudio llevó esta exploración a nivel de dimensiones, donde solo la segunda dimensión (¿Cómo hace usted sus tareas?) y la tercera dimensión (¿Cómo prepara usted sus exámenes?) mostraron asociación significativa. Estos resultados son coherentes toda vez que ambas dimensiones están conectadas con actividades que repercuten directamente en las calificaciones que el alumno obtiene durante sus cursos (tareas y exámenes). Las otras dimensiones no son menos importantes, pues atienden a hábitos vinculados a sus procesos de aprendizaje (el ambiente de estudio, cómo estudia, cómo escucha en clases); sin embargo, en términos de las «calificaciones» asignadas al alumno, las tareas y los exámenes tienen un impacto directo. No obstante, el tamaño de efecto de la segunda y tercera dimensión es también discreto: en promedio, por cada unidad de aumento en el CASM-85 (escala 0 - 53) el alumno ganaría hasta .10 puntos de incremento en su rendimiento académico (calificación 0 - 20). En otras palabras, para que un estudiante tipo mejore 1 punto en sus calificaciones necesitaría mejorar al menos en 10 aspectos distintos de sus hábitos de estudio. Para explicar esta asociación débil, nuestra hipótesis es que los hábitos de estudio son uno de muchos factores que determinan el rendimiento académico, factores que incluyen los estilos de enseñanza (Wentzel, 2002), los estilos de aprendizaje (Akhlaghi, Mirkazemi, Jafarzade, \& Akhlaghi, 2018), la inteligencia (Vega-Hernández, Patino-Alonso, Cabello, Galindo-Villardón, \& Fernández-Berrocal, 2017), nutrición (Burrows, Goldman, Pursey, \& Lim, 2017), la actividad física (Álvarez-Bueno et al., 2017), el nivel socio-económico (Hair, Hanson, Wolfe, \& Pollak, 2015), entre otros. En conclusión, la asociación entre el CASM-85 y el rendimiento académico no es fuerte, pero se encuentra dentro de un rango 
moderado considerando la naturaleza multifactorial de este último.

Hemos detectados fortalezas y limitaciones en nuestro estudio que es preciso declarar. Entre las fortalezas, podemos destacar que este es el primer estudio con el CASM-85 que incluye información de estudiantes del norte, centro y sur del país. En el Perú, este tipo de estudio multicéntrico es aún difícil de coordinar, por lo que su producto tiene un valor especial. Asimismo, la muestra de este estudio $(n=$ 2075) es la más grande reportada hasta la fecha para una revisión psicométrica del CASM-85, muestra que incluye alumnos de ambos sexos provenientes de colegios estatales y particulares. Todas estas fortalezas suman en favor de la representatividad, lo que implica una buena posición para generalizar los resultados. Entre las limitaciones, cabe mencionar que no pudimos acceder a los datos de rendimiento académicos de todos los alumnos de la muestra (solo a un 53\% de ellos); no obstante, el tamaño logrado para ese análisis $(n=1092)$ aún brinda poder suficiente para obtener conclusiones confiables sobre la validez de criterio. No mostramos el análisis de invarianza por grado de estudios; sin embargo, al estar esta variable altamente correlacionada con la edad, los resultados de este análisis fueron similares (idénticas conclusiones sobre la invarianza, los detalles pueden ser solicitados al autor corresponsal). Finalmente, no realizamos ninguna prueba formal con estudiantes para verificar si los ítems inversos del CASM-85 podrían estar afectando la comprensión del sentido de las preguntas; no obstante, nuestra revisión de la redacción de los ítems concluyó que esto sería poco probable.

Los resultados del estudio tienen implicancias prácticas inmediatas, lo mismo que sugiere futuras direcciones en la investigación con el CASM-85. En principio, la forma reducida (45 ítems) presenta mejores propiedades psicométricas y por ello recomendamos su uso; sin embargo, es el usuario quien debe valorar la evidencia disponible y elegir (American Educational Research Association,
American Psychological Association, \& National Council on Measurement in Education, 2014). Para ello, el usuario debería tener presente que nuestro estudio es uno independiente (el autor del CASM-85 no forma parte del equipo) y solo busca brindar información objetiva que lo ayude a decidir. En ese sentido, y para procurar equidad y transparencia, hemos incluido también en este artículo todos los resultados de nuestros análisis con la forma original del CASM-85. Si al final de su evaluación, el usuario opta por la forma reducida, puede escribirnos para proporcionarle las normas de calificación correspondientes. Por otra parte, nuestro contacto con los alumnos durante el estudio nos ha brindado lecciones adicionales que pueden guiar la investigación futura con el CASM-85 o instrumentos similares. Los alumnos de la actual generación incorporan dentro de sus hábitos de estudio elementos tecnológicos que el CASM-85 no toma en cuenta, algunos de ellos muy activos en el proceso de aprendizaje (e.g., Internet) y otros fuertes distractores (e.g., smartphones, videojuegos y redes sociales). Por ello, recomendamos que la futura investigación psicométrica con este y otros instrumentos similares apunte a llenar este «vacío tecnológico», el cual se irá haciendo más generalizado al paso que la tecnología sea más accesible en todas las regiones del interior del país. Por el momento, el CASM-85 sigue siendo un instrumento efectivo y nos queda claro que su autor ya ha empezado a trabajar en la ruta de su actualización (Vicuña, 2014).

En conclusión, el CAMS-85 es un instrumento válido y confiable para evaluar los hábitos de estudio en estudiantes peruanos del norte, centro y sur del país. En especial, su forma reducida de 45 ítems ha mostrado mayor consistencia y validez de estructura interna respecto al modelo de cinco dimensiones propuesto por su autor. Con la forma reducida, esta estructura se mantiene a través de las distintas poblaciones evaluadas. Considerando que con ella también se puede usar una norma de calificación única, la forma reducida facilita las comparaciones entre estas poblaciones. 


\section{Conflicto de intereses}

Los autores declaran no tener conflicto de intereses.

\section{Responsabilidad ética}

El proyecto del estudio ha sido aprobado en forma independiente por el Comité de Ética de PSYCOPERU y el de la Universidad Católica Los Ángeles de Chimbote, ambos bajo la modalidad de exoneración por tratarse de un estudio psicométrico sin mayor impacto en la población evaluada. Las recomendaciones brindadas fueron seguidas, como la posibilidad de disentimiento a la evaluación por parte de cada estudiante evaluado, confidencialidad en el manejo de sus datos, la posibilidad de cada estudiante de acceder a sus propios resultados y el de la institución de solicitar los resultados generales de las evaluaciones realizadas en su centro (anonimizadas). Respecto a las autorizaciones para el uso del instrumento, el manual del CASM-85 (Vicuña, 1998, 2005) no señala ninguna restricción para su uso en investigación. No obstante, contactamos al autor vía correo electrónico $\mathrm{y}$, tras un período de espera prudencial, no recibimos ninguna respuesta negativa de su parte. En tal sentido, procedimos a iniciar este estudio independiente de revisión psicométrica.

\section{Contribución de autoría}

JFQ: Primer borrador del artículo y redacción definitiva del artículo.

JWPC: Trabajo de campo en Lima, primer borrador del artículo y redacción definitiva del artículo.

YCH: Trabajo de campo en Arequipa y redacción definitiva del artículo.
HG: Soporte tecnológico y redacción definitiva del artículo.

RP: Trabajo de campo en Pucallpa y redacción definitiva del artículo.

GGV: Trabajo de campo en Pucallpa y redacción definitiva del artículo.

ACG: Trabajo de campo en Chimbote y redacción definitiva del artículo.

EL: Trabajo de campo en Chimbote y redacción definitiva del artículo.

MC: Trabajo de campo en Chimbote y redacción definitiva del artículo.

JCBA: Autor de la idea original de este estudio y director general del proyecto, a cargo del análisis formal, primer borrador del artículo y redacción definitiva del artículo.

\section{Agradecimientos}

El presente estudio ha sido cofinanciado por PSYCOPERU y la ULADECH-Católica.

Queremos agradecer a los estudiantes, a sus padres $\mathrm{y}$ tutores, y a los directivos de los centros educativos participantes por brindarnos todo el apoyo necesario para la recolección de los datos que hicieron posible este estudio. Agradecimientos especiales para Ida Milla, investigadora asociada de PSYCOPERU, por su colaboración en la coordinación de las labores de campo, lo mismo que a todos los estudiantes de Psicología que apoyaron en dicha tarea. 


\section{Referencias}

Aiken, L. R. (1985). Psychological testing and assessment. US: Allyn \& Bacon.

Akhlaghi, N., Mirkazemi, H., Jafarzade, M., \& Akhlaghi, N. (2018). Does learning style preferences influence academic performance among dental students in Isfahan, Iran? Journal of Educational Evaluation for Health Professions, 15. doi: 10.3352/ jeehp.2018.15.8

Al Shawwa, L., Abulaban, A. A., Abulaban, A. A., Merdad, A., Baghlaf, S., Algethami, A., ... Balkhoyor, A. (2015). Factors potentially influencing academic performance among medical students. Advances in Medical Education and Practice, 6, 65-75. doi: 10.2147/AMEP.S69304

Álvarez-Bueno, C., Pesce, C., Cavero-Redondo, I., Sánchez-López, M., Garrido-Miguel, M., \& MartínezVizcaíno, V. (2017). Academic Achievement and Physical Activity: A Meta-analysis. Pediatrics, 140(6). doi: $10.1542 /$ peds.2017-1498

Alzahrani, S. S., Soo-Park, Y., \& Tekian, A. (2018). Study habits and academic achievement among medical students: A comparison between male and female subjects. Medical Teacher, 40, 1-9. doi: 10.1080/ 0142159X.2018.1464650

American Educational Research Association, American Psychological Association \& National Council on Measurement in Education (2014). Standards for Educational and Psychological Testing. Washington, DC: American Educational Research Association.

Armas, J. J. (2017). Propiedades Psicométricas del Inventario de Hábitos de Estudio CASM-85 revisión 2014 en alumnos de secundaria del Distrito de Huamachuco, 2017 (Tesis de licenciatura). Universidad César Vallejo, La Libertad. Recuperada de http://repositorio.ucv.edu.pe/handle/UCV/651

Atamari, L. F. (2014). Clima social familiar y su relación con los hábitos de estudio en estudiantes de educación intercultural bilingüe de la escuela académico profesional de educación de la Universidad Peruana Unión Filial Juliaca (Tesis de maestría). Universidad Peruana Unión, Lima.
Beauducel, A., \& Herzberg, P. Y. (2006). On the Performance of maximum Likelihood Versus Means and Variance Adjusted Weighted Least Squares Estimation in CFA. Structural Equation Modeling: A Multidisciplinary Journal, 13(2), 186-203. doi: 10.1207/s15328007sem1302_2

Beauducel, A., \& Wittmann, W. W. (2005). Simulation Study on Fit Indexes in CFA Based on Data With Slightly Distorted Simple Structure. Structural Equation Modeling: A Multidisciplinary Journal, 12(1), 41-75. doi: 10.1207/s15328007sem1201_3

Beteta, M. C. (2008). Relación entre la Autoeficacia en el Rendimiento Escolar y los Hábitos de Estudio en el Rendimiento Académico en Alumnos de Secundaria. REICE, 6(3), 59-99. Recuperado de http:/ /www.rinace.net/arts/vol6num3/art3.pdf

Bickerdike, A., O’Deasmhunaigh, C., O’Flynn, S., \& O'Tuathaigh, C. (2016). Learning strategies, study habits and social networking activity of undergraduate medical students. International Journal of Medical Education, 7, 230-236. doi: 10.5116/ijme.576f.d074

Bollen, K. A. (1980). Issues in the Comparative Measurement of Political Democracy. American Sociological Review, 45(3), 370-390. doi: 10.2307/ 2095172

Brown, T. A. (2014). Confirmatory Factor Analysis for Applied Research. New York: Guilford Publications.

Bruna, D., Pérez, M. V., Bustos, C., \& Núñez, J. C. (2017). Propiedades Psicométricas del Inventario de Procesos de Autorregulación del Aprendizaje en Estudiantes Universitarios Chilenos. Revista Iberoamericana de Diagnóstico y Evaluación Psicológica, 44(2), 77-91. doi: 10.21865/ RIDEP44.2.07

Burrows, T., Goldman, S., Pursey, K., \& Lim, R. (2017). Is there an association between dietary intake and academic achievement: a systematic review. Journal of Human Nutrition and Dietetics, 30(2), 117-140. doi: 10.1111/jhn.12407

Cheng-Hsien, L. (2016). Confirmatory factor analysis with ordinal data: Comparing robust maximum likelihood and diagonally weighted least squares. Behavior Research Methods, 48(3), 936-949. doi: 10.3758/s13428-015-0619-7 
Chilca, L. (2017). Autoestima, hábitos de estudio y rendimiento académico en estudiantes universitarios. Propósitos y Representaciones, 5(1), 71-127. doi: 10.20511/pyr2017.v5n1.145

Credé, M., \& Kuncel, N. R. (2008). Study Habits, Skills, and Attitudes: The Third Pillar Supporting Collegiate Academic Performance. Perspectives on Psychological Science, 3(6), 425-453. doi: 10.1111/ j.1745-6924.2008.00089.x

Cronbach, L. J. (1951). Coefficient alpha and the internal structure of tests. Psychometrika, 16(3), 297-334. doi: 10.1007/BF02310555

Cuadros, V. C., Morán, B. V., \& Torres, A. Y. (2017). Hábitos de estudio, disposición hacia el estudio y rendimiento académico en estudiantes de formación profesional técnica de un Instituto armado (Tesis de maestría). Universidad Marcelino Champagnat, Lima. Recuperada de http://repositorio.umch.edu.pe/handle/ $\mathrm{UMCH} / 109$

Cueto, S. (2004). Factores Predictivos del Rendimiento Escolar, Deserción e Ingreso a Educación Secundaria en una Muestra de Estudiantes de Zonas Rurales del Perú. Education Policy Análisis, 12(35). Recuperado de http://repositorio.minedu.gob.pe/handle/ $123456789 / 150$

Diseth, Å., Pallesen, S., Brunborg, G. S., \& Larsen, S. (2010). Academic achievement among first semester undergraduate psychology students: the role of course experience, effort, motives and learning strategies. Higher Education, 59(3), 335-352. doi: 10.1007/ s10734-009-9251-8

DiStefano, C., \& Morgan, G. B. (2014). A Comparison of Diagonal Weighted Least Squares Robust Estimation Techniques for Ordinal Data. Structural Equation Modeling: A Multidisciplinary Journal, 21(3), 425-438. doi: 10.1080/ 10705511.2014 .915373

Enguita, M. F., Martínez, L. M., \& Gómez, J. R. (2010). School Failure and Dropouts in Spain. Madrid: la Caixa.

Freiberg, A., Ledesma, R., \& Fernández, M. M. (2017). Análisis de las Propiedades Psicometricas del Inventario de Estrategias de Aprendizaje y Estudio
(LASSI) en Estudiantes Universitarios. Revista Iberoamericana de Diagnóstico y Evaluación, 44(2), 116-130. doi: 10.21865/RIDEP44.2.10

Hair, N. L., Hanson, J. L., Wolfe, B. L., \& Pollak, S. D. (2015). Association of Child Poverty, Brain Development, and Academic Achievement. JAMA Pediatrics, 169(9), 822-829. doi: 10.1001/ jamapediatrics.2015.1475

Hu, L., \& Bentler, P. M. (1999). Cutoff criteria for fit indexes in covariance structure analysis: Conventional criteria versus new alternatives. Structural Equation Modeling: A Multidisciplinary Journal, 6(1), 1-55. doi: 10.1080/10705519909540118

Huamán, M. I. (2017). Hábitos de estudio en estudiantes de un Centro Preuniversitario de una Universidad Privada de Lima Metropolitana (Tesis de licenciatura). Universidad Inca Garcilaso de la Vega, Lima. Recuperada de http://repositorio.uigv.edu.pe/handle/ 20.500.11818/1376

Huayta, R. S., \& Huayllani, Y. H. (2014). Influencia de los hábitos de estudio en el rendimiento escolar de los estudiantes de primero a quinto grado de educación secundaria en el área de historia, geografía y economía de la institución educativa privada Santiago Ramón y Cajal del distrito de Uchumayo. Universidad Nacional de San Agustín de Arequipa, Arequipa. Recuperado de http:// repositorio.unsa.edu.pe/handle/UNSA/1968

Instituto Nacional de Estadística e Informática (INEI, 2015). Mapa de pobreza provincial y distrital 2013. Lima: INEI.

Instituto Nacional de Estadística e Informática (INEI, 2018). Encuesta Nacional de Programas Presupuestales 2011-2017. Lima: INEI.

Jorgensen, T. D., Pornprasertmanit, S., Schoemann, A. M., \& Rosseel, Y. (2018). semTools: Useful Tools for Structural Equation Modeling. $R$ package version 0.5-1.

Larose, S., Bernier, A., \& Tarabulsy, G. M. (2005). Attachment State of Mind, Learning Dispositions, and Academic Performance During the College Transition. Developmental Psychology, 41(1), 281289. doi: 10.1037/0012-1649.41.1.281 
Lucas, F. Y. (2017). Estilo de aprendizaje y hábitos de estudio asociado al rendimiento académico en la facultad de ciencias administrativas de una universidad nacional Lima (Tesis de maestría). Universidad Cesar Vallejo, Lima. Recuperado de http:/ /repositorio.ucv.edu.pe/bitstream/handle/UCV/12809/ Lucas_OFY.pdf?sequence $=1 \&$ isAllowed $=y$

Manuel, S. P., Grewal, G. K., \& Lee, J. S. (2018). Millennial Resident Study Habits and Factors that Influence American Board of Anesthesiology InTraining Examination Performance: A MultiInstitutional Study. The Journal of Education in Perioperative Medicine: JEPM, 20(2), E623.

Marchesi, A. (2003). El fracaso escolar en España. Madrid: Ediciones doce calles.

Marsh, H. W., Morin, A. J. S., Parker, P. D., \& Kaur, G. (2014). Exploratory Structural Equation Modeling: An Integration of the Best Features of Exploratory and Confirmatory Factor Analysis. Annual Review of Clinical Psychology, 10(1), 85-110. doi: 10.1146/ annurev-clinpsy-032813-153700

Meredith, W. (1993). Measurement invariance, factor analysis and factorial invariance. Psychometrika, 58(4), 525-543. doi: 10.1007/BF02294825

Meza, S. P. (2016). Correlación entre los hábitos de estudio y el rendimiento escolar en los estudiantes del nivel secundario de la Institución Educativa José Teobaldo Paredes Valdez de Arequipa (Tesis doctoral). Universidad Católica de Santa María, Arequipa.

Mira, E., \& López. (1995). Cómo estudiar y cómo aprender. Buenos Aires: Ed. Kapelusz.

Miranda, E. H. (2017). Factores psicosociales y su influencia en el rendimiento académico de los estudiantes de la asignatura de Medicina Estomatológica en la Universidad Privada Antenor Orrego durante el periodo 2013 (Tesis doctoral). Universidad Privada Antenor Orrego, Trujillo.

Osés, R. M., Aguayo, J. C., Duarte, E., \& Manuel, J. I. (2010). Hábitos de estudio y autorregulación. Validación de instrumentos para su medición. Enseñanza e Investigación en Psicología, 15(2), 343-356. Recuperado de https://www.redalyc.org/ articulo.oa?id=29215980007
Pérez, M. G. de Vegas, \& Silva, Z. R. S. (2018). Los hábitos de estudio y rendimiento académico en estudiantes de Enfermería de la Universidad Peruana del Oriente, San Juan Bautista, 2011. Revista de Enfermería UPO, 1(1), 18.

Raykov, T. (2001). Estimation of congeneric scale reliability using covariance structure analysis with nonlinear constraints. The British Journal of Mathematical and Statistical Psychology, 54(2), 315-323. doi: 10.1348/000711001159582

Rebaza, K. F. (2016). Propiedades psicométricas del inventario de hábitos de estudio en estudiantes del nivel secundario de la provincia de Otuzco (Tesis de licenciatura). Universidad César Vallejo, Trujillo. Recuperada de http://repositorio.ucv.edu.pe/handle/ $\mathrm{UCV} / 605$

Reynaga, G. (2009). Mujeres indígenas y la educación superior en el Perú. ISEES: Inclusión Social y Equidad En La Educación Superior, 57-67.

Rosales, J. G. (2016). Estrés académico y hábitos de estudio en universitarios de la carrera de Psicología de un centro de formación superior de privada de Lima-Sur (Tesis de licenciatura). Universidad Autónoma del Perú, Lima.

Salas, O. (2017). Estilos de crianza y hábitos de estudio en estudiantes con violencia familiar de una institución educativa de la ciudad de Piura - 2017 (Tesis de licenciatura). Universidad Cesar Vallejo, Piura. Recuperada de http://repositorio.ucv.edu.pe/ handle/UCV/10712

Salem, R. O., Al-Mously, N., Nabil, N. M., Al-Zalabani, A. H., Al-Dhawi, A. F., \& Al-Hamdan, N. (2013). Academic and socio-demographic factors influencing students' performance in a new Saudi medical school. Medical Teacher, 35(1), S83-S89. doi: 10.3109/ 0142159X.2013.765551

StataCorp (2017). Stata Statistical Software: Release 15. College Station, TX: StataCorp LLC.

Stover, J. B., Uriel, F., \& Fernández, M. (2012). Inventario de Estrategias de Aprendizaje y Estudio: Análisis Psicométrico de una Versión Abreviada. Revista Argentina de Ciencias del Comportamiento, 4(3), 4-12. Recuperado de https://www.redalyc.org/ articulo.oa? $\mathrm{id}=333427358002$ 
Sze, W. W. (2017). Relationships among grit, academic performance, perceived academic failure, and stress in associate degree students. Journal of Adolescence, 60, 148-152. doi: 10.1016/ j.adolescence.2017.08.006

Ubaka, C. M., Sansgiry, S. S., \& Ukwe, C. V. (2015). Cognitive Determinants of Academic Performance in Nigerian Pharmacy Schools. American Journal of Pharmaceutical Education, 79(7), 101. doi: 10.5688/ajpe797101

Vega-Hernández, M. C., Patino-Alonso, M. C., Cabello, R., Galindo-Villardón, M. P., \& Fernández-Berrocal, P. (2017). Perceived Emotional Intelligence and Learning Strategies in Spanish University Students: A New Perspective from a Canonical Non-symmetrical Correspondence Analysis. Frontiers in Psychology, 8, 1888. doi: 10.3389/fpsyg.2017.01888

Vicuña, L. (1985). Inventario de hábitos de estudio CASM-85. Perú: Ediciones CEDEIS.
Vicuña, L. (1998). Inventario de hábitos de estudio CASM 85-R-1998. Perú: Ediciones CEDEIS.

Vicuña, L. (2005). Inventario de hábitos de estudio CASM 85-R-2005. Perú: Grafi-K-Gr.

Vicuña, L. (2014). Inventario de hábitos de estudio CASM 85-R-2014. Peru: Grafi-K-Gr.

Villardón, M. L. (2006). Evaluación del aprendizaje para promover el desarrollo de competencias. Educatio Siglo XXI, 24, 57-76.

Wentzel, K. R. (2002). Are Effective Teachers Like Good Parents? Teaching Styles and Student Adjustment in Early Adolescence. Child Development, 73(1), 287301.

West, C., \& Sadoski, M. (2011). Do study strategies predict academic performance in medical school? Medical Education, 45(7), 696-703. doi: 10.1111/ j.1365-2923.2011.03929.x

\section{Joel Figueroa-Quiñones}

Instituto de Investigación, Universidad Católica los Ángeles de Chimbote (ULADECH-Católica), Chimbote, Perú.

Bachiller en Psicología, Food Addiction in Latin America.

ORCID: 0000-0003-3907-7606

joelfq.13@gmail.com

Juan Walter Pomahuacre Carhuayal

PSYCOPERU Instituto de Investigación, Capacitación y Desarrollo Psicosocial y Educativo, Lima, Perú.

Magister en Psicología Clínica, mención en terapia de niños y adolescentes.

ORCID: 0000-0002-6769-6706

walter.pomahuacre@gmail.com

\section{Yuri Chávez}

Escuela Profesional de Psicología, Universidad Nacional de San Agustín de Arequipa, Arequipa, Perú.

Maestría en ciencias, mención en psicología clínica educativa, infantil y adolescencia.

ORCID: 0000-0003-1857-6094

ychavezps@gmail.com

Heber Gómez

PSYCOPERU Instituto de Investigación, Capacitación y Desarrollo Psicosocial y Educativo, Lima, Perú. Ingeniero en informática y sistemas.

ORCID: 0000-0002-7259-7817

hebergh@gmail.com 


\section{Roxana Portocarrero}

Instituto de Investigación, Universidad Católica los Ángeles de Chimbote (ULADECH-Católica), Chimbote, Perú.

Maestría en Educación.

ORCID: 0000-0002-9061-844X

martina6289@hotmail.com

Giulianna Gamero-Vega

Instituto de Investigación, Universidad Católica los Ángeles de Chimbote (ULADECH-Católica), Chimbote, Perú.

Químico Farmacéutico, Investigación en faith-based interventions y faith-placed interventions de salud: situación actual y perspectivas en Latinoamérica.

ORCID: 0000-0002-5827-9599

igamerov@uladech.edu.pe

Ada Calderón-Giraldo

PSYCOPERU Instituto de Investigación, Capacitación y Desarrollo Psicosocial y Educativo, Lima, Perú.

Licenciada en Psicología.

ORCID: 0000-0002-2068-2542

accg180@gmail.com

Elizabeth López

PSYCOPERU Instituto de Investigación, Capacitación y Desarrollo Psicosocial y Educativo, Lima, Perú.

Maestría en psicología clínica y de la salud.

ORCID: 0000-0002-3762-6109

elizabethlopezrivera@yahoo.es

Martín Castro

PSYCOPERU Instituto de Investigación, Capacitación y Desarrollo Psicosocial y Educativo, Lima, Perú.

Maestría en psicología clínica y de la salud.

ORCID: 0000-0002-8882-6135

martincastros@yahoo.es

\section{Juan Carlos Bazo-Alvarez}

Instituto de Investigación, Universidad Católica los Ángeles de Chimbote (ULADECH-Católica), Chimbote, Perú. PSYCOPERU Instituto de Investigación, Capacitación y Desarrollo Psicosocial y Educativo, Lima, Perú

Doctorando en Investigación Metodológica, Maestro en Investigación Epidemiológica, Especialista en Estadística en Investigación y Estadísticas Educativas, con una amplia experiencia en investigación, en la empresa privada y el Estado, especialmente orientada hacia psicometría y evaluación.

ORCID: 0000-0002-6169-8049

Autor corresponsal: juan.bazo.a@gmail.com 